\title{
Articular Cartilage Degradation in Osteoarthritis
}

\author{
Mary B. Goldring, PhD
}

Received: 26 August 2011/Accepted: 17 November 2011/Published online: 24 January 2012

(C) Hospital for Special Surgery 2012

Keywords osteoarthritis $\cdot$ inflammation $\cdot$ cartilage

\section{Cartilage Composition}

The composition and cellular organization of human adult articular cartilage are complex with qualitative and quantitative differences in matrix constituents ranging from the superficial through deep zones and between the interterritorial and territorial, or pericellular, regions. Chondrocytes, the unique cellular component of articular cartilage, maintain the matrix components under normal, low turnover conditions in which the glycosaminoglycans on proteoglycans and other noncollagen molecules can be replaced. The pericellular matrix is also important, with constituents such as collagen VI, fibromodulin, and matrilin 3 , but little or no type II collagen.

\section{Cartilage Degradation in OA}

During the development of osteoarthritis (OA), the normal, quiescent chondrocytes become activated and undergo a phenotypic shift, resulting in fibrillation and degradation of cartilage matrix, the appearance of chondrocyte clusters, increased cartilage calcification associated with tidemark advancement or duplication, and vascular penetration from the subchondral bone (Fig. 1). Coincident upregulation of

\footnotetext{
M. B. Goldring $\mathrm{PhD}(\square)$

Research Division,

Hospital for Special Surgery,

535 East 70th Street, New York, NY 10021, USA

e-mail: goldringm@hss.edu

M. B. Goldring $\mathrm{PhD}$

BCMB: Cell and Developmental Biology Program,

Weill Cornell Graduate School of Medical Sciences, New York, NY, USA
}

cartilage-degrading proteinases produces matrix degradation products which can further promote catabolic activation, aberrant, hypertrophy-like differentiation, and apoptosis. Once the collagen network is degraded, it cannot be repaired to its original state. Thus, the therapeutic challenge is to prevent the damage or promote repair to recapitulate the physiological and functional properties of the original cartilage.

Risk factors for cartilage loss in osteoarthritis are related either to the adverse effects of trauma or overloading (e.g., injury and obesity) on otherwise normal cartilage or normal loading on abnormal cartilage (e.g., genetic defects and aging), both of which result in abnormal biomechanics and malignment. An inflammatory component also exists, marked by joint pain, swelling, and stiffness, and accompanied by synovitis and the production of inflammatory cytokines, chemokines, adipokines, and other mediators, which can be measured in synovial fluids from arthritic joints.

\section{Activation of Catabolism and Inflammation in the Arthritic Cartilage}

The activation of stress- and inflammation-induced signaling and transcriptional and posttranscriptional events results in release of the chondrocytes from growth arrest, imbalanced homeostasis, and chondrocyte activation with aberrant expression of inflammation-related genes, including nitric oxide synthase-2, cyclooxygenase-2, and catabolic genes such as MMP-1, 3, and 13, and ADAMTS-4 and 5 [5].

The signaling kinases activated by mechanical and inflammatory stimuli include the stress- and mitogenactivated ERK, p38, and JNK, which coordinately activate AP-1, ETS, Runx2, and C/EBP transcription factors, and IKK $\alpha$ and IKK $\beta$, which activate, respectively, noncanonical (RelA/p52) and canonical NF-kB (p65/p50) pathways $[5,11]$. We have identified genes that act in cartilage, 


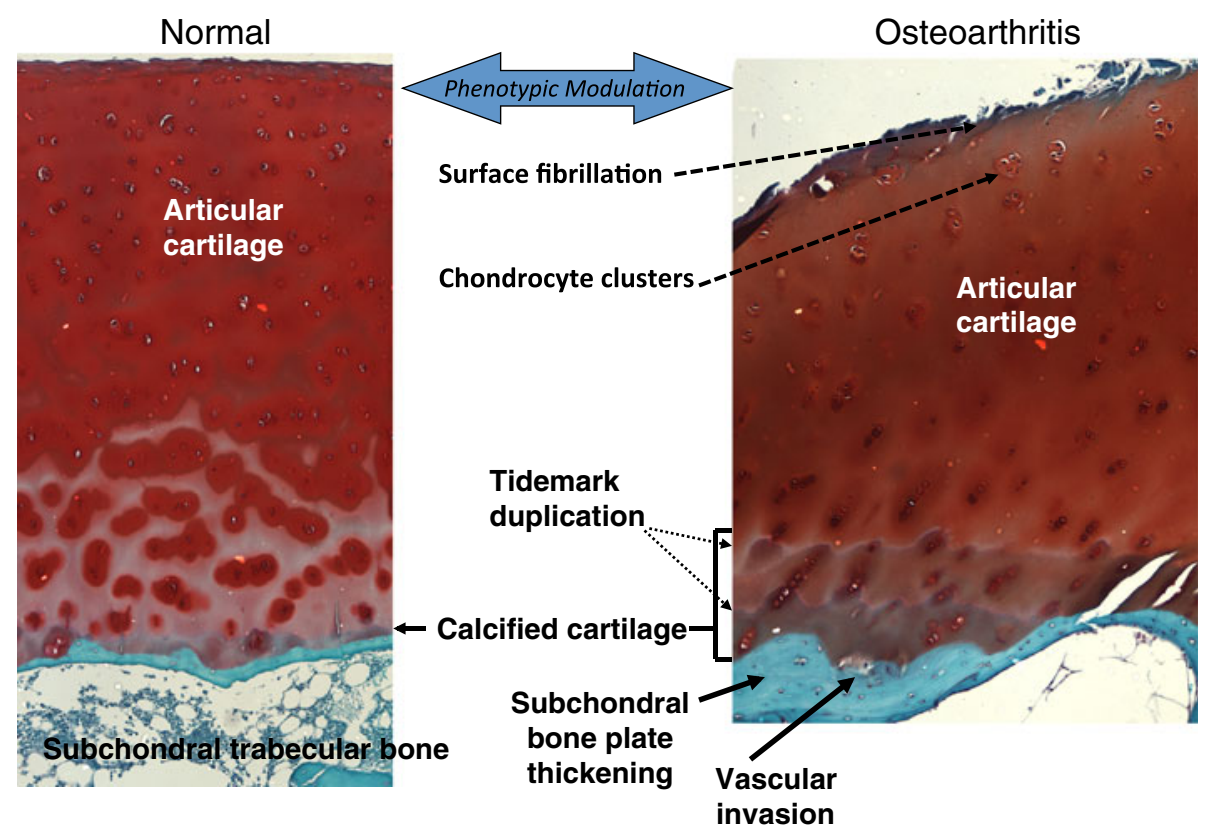

Fig. 1. From homeostasis to osteoarthritis. The composition and cellular organization of healthy human adult articular cartilage (left panel) is more complex than it looks in this histological section showing few chondrocytes distributed in matrix with homogeneous appearance and the clear demarcation (tidemark) between the articular cartilage and a thin zone of calcified cartilage next to the underlying subchondral bone. During the development of osteoarthritis, the normally quiescent chondrocytes become activated and undergo a phenotypic shift, resulting in surface fibrillation and degradation of cartilage matrix, the appearance of chondrocyte clusters, increased cartilage calcification associated with tidemark advancement or duplication, and vascular penetration from the subchondral bone (right panel). Histology (Safranin O/Fast green staining) provided by Cecilia Dragomir, Hospital for Special Surgery, New York, NY; $\times 10$ magnification

including the ETS transcription factor, ESE1/ELF3, induced by inflammatory cytokines, and growth arrest and DNA damage- $45 \beta$, induced in chondrocytes by bone morphogenetic protein-2, both of which are dependent upon NF-KB signaling. Other important mediators in the activation of downstream inflammatory and catabolic events in articular cartilage are the secreted damage-associated molecular patterns or alarmins, including S100A4, A8, A9, and A11, high-mobility group box protein 1 , and other ligands that act through toll-like receptors and receptor for advanced glycation end products (RAGE) [2, 4, 7, 10, 12, 15, 19]. RAGE ligands can also increase reactive oxygen species through upregulating cytokines and chemokines [15]. These events may also cause oxidative stress and chondrocyte apoptosis by altering mitochondrial function [1]. Differential use of the NF- $\mathrm{kB}$ signaling pathways by these ligands may also cause a phenotypic shift of some chondrocytes to expression of hypertrophy-related genes such as COL10A1 and Runx2 [5, 11].

Chondrocytes have receptors for extracellular matrix components, many of which are responsive to mechanical stimulation and are catabolic activators. These include: the integrins, receptors for fibronectin and type II collagen fragments that stimulate expression of proteinases, inflammatory cytokines, and chemokines, as a feedback amplification mechanism once matrix degradation has been established; syndecan-4, a transmembrane heparan sulfate proteoglycan, a positive effector of ADAMTS- 5 activation through controlling MMP-3 synthesis [3]; and discoidin domain receptor 2 (DDR2), a receptor tyrosine kinase that binds specifically to type II and X collagen fibrils and selectively induces MMP-13 expression [17].

\section{Animal Models of OA}

Studies of animal models of post-traumatic osteoarthritis, including transgenic and knockout mice subjected to surgical osteoarthritis [9], have shown the importance of proteoglycan depletion in cartilage erosion as in Adamts 5 knockout mice, which are protected against progressive cartilage loss. However, aggrecan depletion by itself does not drive cartilage erosion, as shown in recent studies in Mmp13 knockout mice, which are protected against cartilage erosion, while aggrecan depletion continues [8]. Knockout of HIF2 $\alpha$, induced by NF- $\mathrm{kB}$ signaling and implicated in cytokine-induced MMP-13, production also protects against osteoarthritis progression [18].

Genetic models with abnormal composition and structure of articular cartilage or other joint tissues may develop spontaneous or accelerated osteoarthritis due to altered biomechanics. Colllal+/- (Cho/+) or Col9a1-/- mice have accelerated disease, which is attenuated, along with reduced MMP-13 expression, when they are crossed with DDR2 \pm mice [17]. This may be due to activity of the serine proteinase, HTRA1, on disrupting the pericellular matrix, composed of matrilin 3, fibronectin, biglycan, fibromodulin, COMP, and collagen VI microfibrils, thereby exposing DDR2 to activation by type II collagen fibrils that are normally not in contact with the chondrocyte [14]. 
Epigenetic mechanisms, involving changes in DNA methylation status at $\mathrm{CpG}$ sites, modifications of histone tails by histone deacetylases, and changes in chromatin structure, may be associated with the degradative phenotype in osteoarthritic cartilage [6]. How specific microRNAs could be influencing the cartilage homeostasis and disease mechanisms has also been receiving greater attention [13].

\section{Summary}

Common mediators determine initiation and progression of cartilage damage across various models of osteoarthritis that can also be observed in the human form of the disease. Work during the past years has uncovered interactions among these mediators that impact the disease process and may inform us about new directions for targeted therapies [16].

Acknowledgments Dr. Goldring's research is supported in part by National Institutes of Health Grants R01-AG022021, R21-AR054887, and RC4 AR060546.

Disclosure The author certifies that she has no commercial associations (e.g., consultancies, stock ownership, equity interest, patent/licensing arrangements, etc.) that might pose a conflict of interest in connection with the submitted article.

\section{References}

1. Blanco FJ, Rego I, Ruiz-Romero C. The role of mitochondria in osteoarthritis. Nat Rev Rheumatol 2011;7:161-169.

2. Campo GM, Avenoso A, Campo S, D'Ascola A, Nastasi G, Calatroni A. Molecular size hyaluronan differently modulates tolllike receptor-4 in LPS-induced inflammation in mouse chondrocytes. Biochimie 2010;92:204-215.

3. Echtermeyer F, Bertrand J, Dreier R, Meinecke I, Neugebauer K, Fuerst M, Lee YJ, Song YW, Herzog C, Theilmeier G, Pap T. Syndecan-4 regulates ADAMTS-5 activation and cartilage breakdown in osteoarthritis. Nat Med 2009;15:1072-1076.

4. Garcia-Arnandis I, Guillen MI, Gomar F, Pelletier JP, MartelPelletier J, Alcaraz MJ. High mobility group box 1 potentiates the pro-inflammatory effects of interleukin-1beta in osteoarthritic synoviocytes. Arthritis Res Ther 2010;12:R165.

5. Goldring MB, Otero M, Plumb DA, Dragomir C, Favero M, El Hachem K, Hashimoto K, Roach HI, Olivotto E, Borzi RM, Marcu KB. Roles of inflammatory and anabolic cytokines in cartilage metabolism: signals and multiple effectors converge upon MMP-13 regulation in osteoarthritis. Eur Cell Mater 2011;21:202-220.
6. Hashimoto K, Oreffo RO, Gibson MB, Goldring MB, Roach HI. DNA demethylation at specific $\mathrm{CpG}$ sites in the IL1B promoter in response to inflammatory cytokines in human articular chondrocytes. Arthritis Rheum 2009;60:3303-3313.

7. Heinola T, Kouri VP, Clarijs P, Ciferska H, Sukura A, Salo J, Konttinen YT. High mobility group box-1 (HMGB-1) in osteoarthritic cartilage. Clin Exp Rheumatol 2010;28:511-518.

8. Little CB, Barai A, Burkhardt D, Smith SM, Fosang AJ, Werb Z, Shah M, Thompson EW. Matrix metalloproteinase 13-deficient mice are resistant to osteoarthritic cartilage erosion but not chondrocyte hypertrophy or osteophyte development. Arthritis Rheum 2009;60:3723-3733.

9. Little CB, Fosang AJ. Is cartilage matrix breakdown an appropriate therapeutic target in osteoarthritis-insights from studies of aggrecan and collagen proteolysis? Curr Drug Targets 2010;11:561-575.

10. Liu-Bryan R, Terkeltaub R. Chondrocyte innate immune myeloid differentiation factor 88-dependent signaling drives procatabolic effects of the endogenous Toll-like receptor 2/Toll-like receptor 4 ligands low molecular weight hyaluronan and high mobility group box chromosomal protein 1 in mice. Arthritis Rheum 2010;62:2004-2012.

11. Marcu KB, Otero M, Olivotto E, Borzi RM, Goldring MB. NFkappaB signaling: multiple angles to target OA. Curr Drug Targets 2010;11:599-613.

12. Miranda KJ, Loeser RF, Yammani RR. Sumoylation and nuclear translocation of S100A4 regulate IL-1beta-mediated production of matrix metalloproteinase-13. J Biol Chem 2010;285:31517-31524.

13. Miyaki $\mathrm{S}$, Sato $\mathrm{T}$, Inoue $\mathrm{A}$, Otsuki $\mathrm{S}$, Ito $\mathrm{Y}$, Yokoyama $\mathrm{S}$, Kato Y, Takemoto F, Nakasa T, Yamashita S, Takada S, Lotz MK, Ueno-Kudo H, Asahara H. MicroRNA-140 plays dual roles in both cartilage development and homeostasis. Genes Dev 2010;24:1173-1185.

14. Polur I, Lee PL, Servais JM, Xu L, Li Y. Role of HTRA1, a serine protease, in the progression of articular cartilage degeneration. Histol Histopathol 2010;25:599-608.

15. Rasheed Z, Akhtar N, Haqqi TM. Advanced glycation end products induce the expression of interleukin- 6 and interleukin8 by receptor for advanced glycation end product-mediated activation of mitogen-activated protein kinases and nuclear factor$\mathrm{KB}$ in human osteoarthritis chondrocytes. Rheumatology (Oxford) 2011;50(5):838-851.

16. van den Berg WB. Osteoarthritis year 2010 in review: pathomechanisms. Osteoarthritis Cartilage 2011;19:338-341.

17. Xu L, Servais J, Polur I, Kim D, Lee PL, Chung K, Li Y. Attenuation of osteoarthritis progression by reduction of discoidin domain receptor 2 in mice. Arthritis Rheum 2010;62:2736-2744.

18. Yang S, Kim J, Ryu JH, Oh H, Chun CH, Kim BJ, Min BH, Chun JS. Hypoxia-inducible factor-2alpha is a catabolic regulator of osteoarthritic cartilage destruction. Nat Med 2010;16:687-693.

19. Zreiqat H, Belluoccio D, Smith MM, Wilson R, Rowley LA, Jones K, Ramaswamy Y, Vogl T, Roth J, Bateman JF, Little CB. S100A8 and S100A9 in experimental osteoarthritis. Arthritis Res Ther 2010;12:R16. 